\title{
The effects of supportive-expressive dynamic psychotherapy on the outcomes of bipolar disorder in men
}

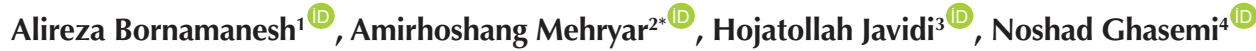 \\ ${ }^{1} \mathrm{PhD}$ Student of Psychology, Department of Psychology, Marvdasht Branch, Islamic Azad University, Marvdasht, Iran \\ ${ }^{2}$ Professor in Psychology, Department of Psychology, Marvdasht Branch, Islamic Azad University, Marvdasht, Iran \\ ${ }^{3}$ Professor in Psychology, Department of Psychology, Marvdasht Branch, Islamic Azad University, Marvdasht, Iran \\ ${ }^{4}$ Assistant Professor, Department of Psychology, Marvdasht Branch, Islamic Azad University, Marvdasht, Iran
}

\begin{abstract}
Background and aims: Bipolar disorder (BD) is a chronic disorder with high recurrence rate and significant disability. BD is refractory to treatments and afflicted patients experience disease recurrence despite medication therapy. Therefore, appropriate psychosocial interventions are needed to improve the effectiveness of medication therapy and facilitate BD management. The aim of this study was to evaluate the effects of supportive-expressive dynamic psychotherapy (SEDP) on the outcomes of BD in men.

Methods: This double-blind randomized clinical study was conducted in 2017-2018 using a two-group pretest-posttest design. Participants were thirty hospitalized men with BD conveniently selected from Khorshid hospital, Isfahan, Iran, and randomly assigned to an intervention $(n=15)$ and a control $(n=15)$ group. Participants in the control group received conventional medication therapy, while participants in the intervention group received conventional medication therapy as well as SEDP in twelve 60-minute one-toone sessions. Participants in both groups completed the Bipolar Depression Rating Scale before, immediately after, three months after, and six months after the intervention. The SPSS program for Windows (v. 23.0) was employed to analyze the data via the analysis of covariance and the repeated measures analysis of variance. Results: Although the mean score of bipolar depression significantly decreased at the first posttest, the between-group difference was not significant $(P>0.05)$. However, the mean score of bipolar depression in the intervention group was significantly less than the control group at the second and the third posttests $(P$ $<0.05)$.

Conclusion: SEDP is effective in significantly reducing mood symptoms among men with BD.

Keywords: Bipolar disorder, Supportive-expressive dynamic psychotherapy, Mood symptoms
\end{abstract}

\author{
*Corresponding Author: \\ Prof. Amirhoshang Mehryar, \\ Professor in Psychology, \\ Department of Psychology, \\ Marvdasht Branch, Islamic \\ Azad University, Marvdasht, \\ Iran. \\ Tel: +987143311150 , \\ Email: amirmehryar36@gmail. \\ com
}

\section{Received: 17 February 2021}

Accepted: 16 April 2021

ePublished: 30 September 2021

\section{Introduction}

Bipolar disorder (BD) is a chronic disorder with high recurrence rate and significant disability (1). There are different types of $\mathrm{BD}$ including $\mathrm{BD}$ I and $\mathrm{BD}$ II. According to the Diagnostic and Statistical Manual of Mental Disorders (DSM-5), at least one lifelong mania is required to diagnose BD I and at least one period of major depression and hypomania is required to diagnose BD II. Clinical cases show that BD II is more common in women, while the prevalence of BD I in men and women is almost equal (2). BD consists of a series of signs and symptoms that last for weeks or months and cause certain changes in functioning (3). In the manic period, patients experience symptoms such as increased self-esteem or arrogance, low need for sleep, abnormal talkativeness, hyperactivity, distraction, increased goal-directed sexual, occupational, academic, or social activities, psychomotor agitation (resulting in purposeless or useless activities), and excessive involvement in activities with high potential of negative consequences (such as wastefulness, sexual indiscretions, or foolish business investments) (4). During the period of depression, symptoms may include depressed mood most of the day and night, marked decrease in interests and pleasures, significant weight loss, insomnia or oversleeping, psychomotor agitation or slowness, fatigue or loss of energy, feeling of worthlessness or excessive guilt, difficulty in thinking or concentration, indecisiveness, recurrent thoughts about death, frequent suicidal thoughts without a specific plan, and suicide attempts or a certain plan for suicide (5).

As rapid mood swings and impulsivity are key factors in suicide attempt and substance abuse disorders among patients with $\mathrm{BD}$, it is essential to use strategies to reduce mood swings and impulsivity. Certainly, the main treatment for $\mathrm{BD}$ is medication therapy. Lithium is the most effective medication for BD. It reduces mood swings, impulsivity, and aggressive behaviors (6). Nonetheless, studies show that BD is refractory to treatments and patients with $\mathrm{BD}$ still experience disease recurrence despite medication therapy $(7,8)$. Therefore, appropriate psychosocial interventions are needed to improve the effectiveness of medication therapy. Selecting the best treatment option is the right of patients and helps save time and healthcare resources.

Previous studies showed that psychotherapy improves the outcomes of $\mathrm{BD}$ treatment $(7,9)$. Dynamic

(C) 2021 The Author(s); Published by Shahrekord University of Medical Sciences. This is an open-access article distributed under the terms of the Creative Commons Attribution License (http://creativecommons.org/licenses/by/4.0), which permits unrestricted use, distribution, and reproduction in any medium, provided the original work is properly cited. 
psychotherapy interventions, such as supportiveexpressive dynamic psychotherapy (SEDP), are mainly based on psychoanalytic theories about the underlying causes of mental disorders and focus on self-expression and awareness of painful memories and emotions (10). Dynamic psychotherapy focuses on unconscious emotions that lead to unconscious anxiety, psychological symptoms, and different defenses and has potential positive effects on patients with multiple psychiatric disorders and patients with mood disorders (11). One of the key components of dynamic psychotherapy is the therapist's continuous attempt to create a deep emotional experience as a healing element (12). The active involvement of the therapist and the correct use of dynamic psychotherapy techniques help clients identify the depth of their emotions and thoughts and further develop their mental health in short periods of time (13).

Studies show that dynamic psychotherapy promotes medication adherence, strengthens patients' motivation to continue treatments, positively affects lifestyle medication, improves social functioning among patients with mental disorders, reduces impulsivity and mood swings, and enhances self-control among patients with BD $(14,15)$. Short-term dynamic psychotherapy has received serious attention in recent years. This type of dynamic psychotherapy is usually the best method to achieve longterm recovery and has significant effects on all aspects of afflicted patients' lives (13). Adequate evidence exists regarding the positive effects of SEDP, a type of short-term dynamic psychotherapy, on mental disorders. Different studies in the last two decades showed that self-expression, either verbal or written, can improve physical and mental health as well as the functioning of the immune and the autonomic nervous systems $(16,17)$.

Despite the known positive effects of dynamic psychotherapy, our literature search revealed that no study had yet evaluated the effects of SEDP on male patients with BD. Therefore, the present study was designed and conducted to reduce this gap. The aim of this study was to evaluate the effects of SEDP on the outcomes of BD in men.

\section{Methods}

\section{Design}

This double-blind randomized clinical study was conducted from March 2017 to September 2018.

\section{Participants and setting}

Participants were thirty male patients with BD hospitalized in Khorshid hospital, Isfahan, Iran. Inclusion criteria were definite diagnosis of $\mathrm{BD}$ by a psychiatrist based on the DSM-5 criteria, hospitalization, age of 20-50 years, basic literacy skills, fluency in Persian, no serious comorbid physical problems (such as sensory impairment or physical disability), no cognitive impairment that altered understanding of and participation in treatment, no serious psychiatric disorders or psychotic symptoms based on the Structured Clinical Interview for DSM-5, no participation in psychological programs during the last six months, and agreement for participation. Exclusion criteria were reluctance to stay in the study, more than one absence from the intervention sessions along with non-participation in the subsequent remedial session, and discharge from the study setting during the study. An invitation announcement was issued in the study setting and volunteered patients were randomly assigned to an intervention $(n=15)$ and a control $(n=15)$ group.

\section{Intervention}

Participants in the control group received routine care services, conventional medication therapy, while their counterparts in the intervention group received conventional medication therapy as well as SEDP in twelve sixty-minute one-to-one sessions. Psychological outcome assessment in the control group was performed at the beginning of the study, immediately after psychological interventions, three months after the pretest, and six months after the pretest. In the intervention group, outcomes were assessed before, immediately after, three months after, and six months after the study intervention.

\section{Instruments}

Study instruments were the research version of the Structured Clinical Interview for DSM-5 Axis I Disorders (SCID-V-RV) as well as the Bipolar Depression Rating Scale (BDRS). The interview, developed by First et al. (18), is a semi-structured interview that helps establish diagnosis based on the DSM-5. Mohammadkhani and Ebrahimzadeh Mousavi translated this interview into Persian (19). This interview was used in the present study to assess the eligibility criteria of non-affliction by serious psychiatric disorders or psychotic symptoms.

BDRS is the first BD-specific instrument with acceptable validity and reliability. A previous study reported that its Cronbach's alpha was 0.81 and the coefficients of the correlation of its score with the scores of the Center for Epidemiological Studies Depression Scale and the Young Mania Rating Scale were 0.78 and 0.82, respectively (20). Another study confirmed the acceptable reliability of the scale with a Cronbach's alpha of 0.81 and its acceptable validity through the coefficients of correlation with the Beck Depression Inventory and the Hamilton Depression Rating Scale of 0.61 and 0.71 , respectively (21).

\section{Data analysis}

The SPSS program for Windows (v. 23.0) was used for data analysis. Data were summarized using the measures of descriptive statistics, namely mean and standard deviation. Moreover, the repeated measures analysis of variance was used to assess the variations of the mean score of BDRS across the four measurement time points.

\section{Results}

A total of 30 male patients with $\mathrm{BD}$ participated in this 
study. The mean of participants' age was $36.76 \pm 8.81$ in the intervention group and 37.01 \pm 9.19 in the control group.

The mean score of BDRS reduced in both groups across the four measurement time points. The analysis of covariance and the repeated measures (Table 1) analysis of variance were used to determine whether these decreases were significant. The analysis of covariance revealed no significant difference between the groups in terms of the mean score of BDRS at the first posttest, i.e., immediately after the intervention $(P=0.197)$ (Table 2). Then, the repeated measures analysis of variance was used to assess the effects of SEDP at the second posttest (i.e., three months after the intervention). The results of the Mauchly's test illustrated that sphericity was assumed (Table 3 ). The intergroup analysis revealed the significant effects of SEDP at the second posttest so that $24 \%$ of the variance of changes in BDRS mean score was explained by the group variable (Table 4). A statistical power of 0.97 also indicated that the number of participants was adequate for this model. Subsequently, the repeated measure analysis of variance was used to assess the effects of the intervention at the third posttest (i.e., six months after the intervention). The Mauchly's test revealed that sphericity was not assumed (Table 5) and hence, the Greenhouse-Geisser correction was used. The inter-group analysis showed that SEDP had significant positive effects on BDRS mean score even at six months after the intervention $(P<0.05)$ (Table 6).

\section{Discussion}

The aim of this study was to evaluate the effects of SEDP on the outcomes of $\mathrm{BD}$ in men. Findings revealed no significant difference between the intervention and the control groups respecting the mean score of BDRS at the first posttest because BDRS mean score reduced in both groups. The decrease in BDRS mean score is attributable to conventional medication therapy in the control group and SEDP in the intervention group. Previous studies highlighted that the positive effects of psychoanalytic therapies appear over time, usually after three months $(21,22)$, because changes in lifestyle behaviors usually need time (23). However, our findings showed that SEDP had significant positive effects on BDRS mean score at three and six months after the intervention and the between-group difference respecting the BDRS mean score was statistically significant at these two time points. These findings are consistent with the findings of several previous studies $(21,24,26)$. The positive effects of SEDP are mainly attributable to its interpretive components

Table 1. The mean score of bipolar depression in the intervention and the control groups across the four measurement time points

\begin{tabular}{|c|c|c|c|c|c|c|c|c|c|}
\hline \multirow{3}{*}{ Variable } & \multirow{3}{*}{ Group } & \multicolumn{8}{|c|}{ Time } \\
\hline & & \multicolumn{2}{|c|}{ Before } & \multicolumn{2}{|c|}{ After } & \multicolumn{2}{|c|}{ Three months after } & \multicolumn{2}{|c|}{ Six months after } \\
\hline & & Mean & SD & Mean & SD & Mean & SD & Mean & SD \\
\hline \multirow{2}{*}{$\begin{array}{l}\text { Bipolar } \\
\text { depression }\end{array}$} & Intervention & 47.53 & 4.75 & 35.67 & 5.23 & 24.53 & 3.85 & 14.80 & 3.34 \\
\hline & Control & 47 & 4.92 & 36.53 & 6.45 & 29.67 & 4.92 & 21.93 & 5.91 \\
\hline
\end{tabular}

Table 2. The results of the analysis of covariance for the effects of supportive-expressive dynamic psychotherapy on bipolar depression immediately after the intervention

\begin{tabular}{ccccccc}
\hline Source of change & Sum squares & Degrees of freedom & Mean squares & F value & $\boldsymbol{P}$ value & Impact \\
\hline Group & 15.27 & 1 & 15.27 & 1.75 & 0.197 & 0.061 \\
Error & 235.50 & 27 & 8.72 & & & \\
\hline
\end{tabular}

Table 3. The results of the Mauchly's test for the equality of covariance

\begin{tabular}{lcccc}
\hline Item & Mauchly's test & Chi-square test & Degrees of freedom & $\boldsymbol{P}$ value \\
\hline Bipolar depression & 0.828 & 5.10 & 2 & 0.078 \\
\hline
\end{tabular}

Table 4. The results of the inter-group effect analysis

\begin{tabular}{|c|c|c|c|c|c|c|c|c|}
\hline Item & & Sum squares & Degrees of freedom & Mean squares & F value & $P$ value & Impact & Power \\
\hline \multirow{2}{*}{$\begin{array}{l}\text { Bipolar } \\
\text { depression }\end{array}$} & Time*Group interaction & 130.69 & 2 & 65.34 & 9.19 & 0.0005 & 0.247 & 0.97 \\
\hline & error & 398.09 & 56 & 7.109 & & & & \\
\hline
\end{tabular}

Table 5. The results of the Mauchly's test for the equality of covariance

\begin{tabular}{|c|c|c|c|c|}
\hline Item & Mauchly's test & Chi-square test & Degrees of freedom & $P$ value \\
\hline Bipolar depression & 0.296 & 32.49 & 5 & 0.0001 \\
\hline
\end{tabular}

Table 6. The results of the inter-group effect analysis

\begin{tabular}{llccccccc}
\hline Item & & Sum squares & Degrees of freedom & Mean squares & F value & $\boldsymbol{P}$ value & Impact & Power \\
\hline $\begin{array}{l}\text { Bipolar } \\
\text { depression }\end{array}$ & Time*Group interaction & 289.36 & 1.70 & 170.74 & 8.73 & 0.0005 & 0.24 \\
\cline { 2 - 8 } & error & 928.33 & 47.45 & 19.56 & & & \\
\hline
\end{tabular}


What does this paper contribute to the wider global clinical community?

SEDP can be effective in improving mood symptoms among patients with BD.

The use of SEDP can reduce the need for medication therapy and the risk of medication side effects.

which improve hope and motivation for emotional and behavioral modifications. A study reported that the greatest effects of SEDP were on patients with moderate to severe psychiatric symptoms (24). Our participants also had moderate to severe symptoms and hence, the significant effects of the study intervention might positively have been affected by symptom severity. The results of a metaanalysis also showed that the greatest effects of dynamic psychotherapy were on target problems and general psychiatric symptoms (27). Another study also reported the relative effectiveness of SEDP in alleviating psychiatric symptoms and facilitating overall recovery (10). Moreover, a study reported that dynamic conflict-based psychotherapy focused on communication significantly reduced psychological problems and dysphoric mood through reducing conflicts (28). Similarly, a study found that grief-based emotional processing was effective in reducing grief and had positive effects on adjustment (29).

The goal of SEDP is to address conflictual relationship and reduce symptoms and eliminate important, but limited, changes in personality. In SEDP, patients find the childhood roots of their problems and thereby, understand that others' responses derive from a transference distortion or a repetition compulsion. Transference distortion holds that individuals experience in their relationships the same feelings they experienced in their relationships with their parents during childhood. Repetition compulsion also means that in their current relationships, individuals stimulate others so that the same behavioral patterns of their parents repeat. For example, they make others angry to shout at them and then, respond by withdrawing from them. SEDP helps patients understand that transference distortion and repetition compulsion negatively affect their interpersonal relationships and reduce their satisfaction with relationships (30).

Unconscious conflicts also play significant role in explaining the psychopathology of mental disorders. Conflicts can be associated with negative psychological outcomes ranging from apathy, anxiety, and isolation to maladaptive aggressive behavioral responses, overobedience, physiological responses, and physical problems (31,32). During psychotherapy sessions, patients internalize the relationship of current behaviors with previous childhood experiences based on the actions of the therapist such as the transformation of unconscious processes into conscious processes. Subsequently, application of the internalized thoughts and beliefs helps clients reduce intrapersonal and interpersonal conflicts and thereby, positively affects their satisfaction with relationships. On the other hand, conflictual relationship focuses on emotions and feelings, particularly on their use in communication. Accordingly, emotions are identified and awareness of unconscious processes is acquired. Therefore, elimination of conflicts seems to reduce sensitivity in relationships and thereby, reduces mood symptoms (33).

\section{Conclusion}

This study shows the effectiveness of SEDP in reducing mood symptoms among men with BD. Therefore, SEDP can be used in combination with medication therapy to reduce mood symptoms, improve functioning, and facilitate recovery among patients with mood disorders.

\section{Limitations}

This study was conducted on men with $\mathrm{BD}$ with an age range of 20-50. Therefore, findings may not easily be generalizable to women with $\mathrm{BD}$ and patients in other age groups. Moreover, we could not monitor the long-term effects of SEDP.

\section{Acknowledgement}

We would like to thank participants of the study.

\section{Conflict of interests}

There is no conflict of interests.

\section{Ethical Approval}

The Ethics Committee of Shiraz Islamic Azad University approved this study (code: IR.IAU.SHIRAZ.REC.1398.002). Moreover, the study was registered in the Iranian Registry of Clinical Trials (code: IRCT20180716040485N1). Data were collected and managed anonymously.

\section{References}

1. Miziou S, Tsitsipa E, Moysidou S, Karavelas V, Dimelis D, Polyzoidou V, et al. Psychosocial treatment and interventions for bipolar disorder: a systematic review. Ann Gen Psychiatry. 2015;14:19. doi: 10.1186/s12991-015-0057-z.

2. American Psychiatric Association. Diagnostic and statistical manual of mental disorders. 5th ed. Translated By: Rezaee, Fakhraee, Farmand, Niloufari, Hashemi Azar, Shamloo. Tehran: Arjmand; 2016. p. 165, 179, 166, 167. [Persian].

3. Feki I, Moalla M, Baati I, Trigui D, Sellami R, Masmoudi J. Impulsivity in bipolar disorders in a Tunisian sample. Asian J Psychiatr. 2016;22:77-80. doi: 10.1016/j.ajp.2016.05.005.

4. Masaki C, Sharpley AL, Cooper CM, Godlewska BR, Singh $\mathrm{N}$, Vasudevan SR, et al. Effects of the potential lithiummimetic, ebselen, on impulsivity and emotional processing. Psychopharmacology (Berl). 2016;233(14):2655-61. doi: 10.1007/s00213-016-4319-5.

5. Barry J. Environment and Social Theory. Routledge; 1999. p. 239.

6. Kakko J, Svanborg KD, Kreek MJ, Heilig M. 1-year retention and social function after buprenorphine-assisted relapse prevention treatment for heroin dependence in Sweden: a randomised, placebo-controlled trial. Lancet. 2003;361(9358):662-8. doi: 10.1016/s0140-6736(03)12600-1.

7. Nayyeri M, Rajaee A, Meschi F, Sodagar S. Effectiveness of dynamic-supportive psychotherapy on type 2 diabetes patients' depression. J North Khorasan Univ Med Sci. 2019;11(3):73-7. doi: 10.21859/nkjmd-110310. [Persian].

8. Rounsaville B, Carroll K, Back S. Individual psychotherapy. In: 
Lowinson JH, Ruize P, Millman R, Langrod J, eds. Substance Abuse: A Comprehensive Texbook. Baltimore: Lippincott Williams \& Wilkins; 2005. p. 653-70.

9. Erfan A, Ghezelbash S, Kazemian M, Noorbala A. The effectiveness of emotional schema therapy on impulsivity and mood symptoms of women with bipolar disorder. J Res Behav Sci. 2019;17(3):388-99. [Persian].

10. Kazemi-Zahrani H, Mohagheghian M. Efficacy of mindfulness based on cognitive therapy on impulsivity and self-inhibition in patients with bipolar disorder under medical treatment. J Shahid Sadoughi Univ Med Sci. 2019;27(7):1701-13. doi: 10.18502/ssu.v27i7.1930. [Persian].

11. Spitzer RL, Williams JB, Gibbon M, First MB. The structured clinical interview for DSM-III-R (SCID). I: history, rationale, and description. Arch Gen Psychiatry. 1992;49(8):624-9. doi: 10.1001/archpsyc.1992.01820080032005.

12. Pennebaker JW, Smyth JM. Opening Up by Writing It Down: How Expressive Writing Improves Health and Eases Emotional Pain. Guilford Publications; 2016. p. 1-216.

13. Abbass A, Sheldon A, Gyra J, Kalpin A. Intensive shortterm dynamic psychotherapy for DSM-IV personality disorders: a randomized controlled trial. J Nerv Ment Dis. 2008;196(3):211-6. doi: 10.1097/NMD.0b013e3181662ffo.

14. Leiper R, Maltby M. Sage Therapeutic Change Series: The Psychodynamic Approach to Therapeutic Change. London: SAGE Publications; 2004.

15. Fathali Lavasani F, Atef Vahid MK, Asgharnejad Farid AA, Farzad V. The effectiveness of supportive-expressive dynamic psychotherapy in improving the treatment outcome for drug dependency. Contemporary Psychology. 2010;4(2):16-24. [Persian]

16. Anbari Meybodi N, Basharpoor S, Aghajani S, Bina E. The effect of psycho-educational intervention on improving ego imparement, human representation and indicators in patients with bipolar disorder. Shenakht Journal of Psychology and Psychiatry. 2019;6(5):96-107. [Persian].

17. Johansson R, Town JM, Abbass A. Davanloo's intensive shortterm dynamic psychotherapy in a tertiary psychotherapy service: overall effectiveness and association between unlocking the unconscious and outcome. PeerJ. 2014;2:e548. doi: 10.7717/peerj.548.

18. First MB, Williams JB, Karg RS, Spitzer RL. Structured Clinical Interview for DSM-5-Research Version (SCID-5 for DSM5, Research Version; SCID-5-RV). Arlington, VA: American Psychiatric Association. 2015.

19. Mohammadkhani P, Ebrahimzadeh Mousavi M. Structured Clinical Interview to Assess SCID-5-RV Disorders: DSM-5 (Research Version). 2017;(1):1-602.

20. Hakim Shooshtari M, Davari-Ashtiani R, Shahrivar Z, Shabani A, Semnani $Y$, Kaviani $H$, et al. Structured clinical interview for DSM-IV (SCID Persian translation and cultural adaptation). Iran J psychiatry. 2007;2(1):46-8.

21. Ebrahimi A, Barekatain M, Bornamanesh A, Nassiri $H$. Psychometric properties of the Persian version of bipolar depression rating scale (BDRS) in patients and general population. Iran J psychiatry Clin Psychol. 2015;21(1):60-8.
22. Barnes E, Simpson S, Griffiths E, Hood K, Craddock N, Smith DJ. Developing an online psychoeducation package for bipolar disorder. J Ment Health. 2011;20(1):21-31. doi: 10.3109/09638237.2010.525565.

23. Mohammadkhani P, Forouzan AS, Hooshyari Z, Abasi I. Psychometric properties of Persian version of structured clinical interview for DSM-5-research version (SCID-5-RV): a diagnostic accuracy study. Iran J Psychiatry Behav Sci. 2020;14(2):e100930. doi: 10.5812/ijpbs.100930.

24. Luborsky L, Woody GE, Hole AV, Velleco A. Supportiveexpressive dynamic psychotherapy for treatment of opiate drug dependence. In: Barber JP, Crits-Christoph $\mathrm{P}$, eds. Dynamic Therapies for Psychiatric Disorders (Axis I). Basic Books; 1995. p. 131-60.

25. Leichsenring F, Kruse J, Rabung S. Efficacy of psychodynamic psychotherapy in specific mental disorders: an update. In: Luyten P, Mayes LC, Fonagy P, Target M, Blatt SJ, eds. Handbook of Psychodynamic Approaches to Psychopathology. The Guilford Press; 2015. p. 485-511).

26. Leibovich L, Front O, McCarthy KS, Zilcha-Mano S. How do supportive techniques bring about therapeutic change: the role of therapeutic alliance as a potential mediator. Psychotherapy (Chic). 2020;57(2):151-9. doi: 10.1037/pst0000253.

27. Leichsenring F, Rabung S, Leibing E. The efficacy of short-term psychodynamic psychotherapy in specific psychiatric disorders: a meta-analysis. Arch Gen Psychiatry. 2004;61(12):1208-16. doi: 10.1001/archpsyc.61.12.1208.

28. Faramarzi M, Azadfallah P, Rasoulzadeh Tabataei K, Shekari Shirvani J. The effect of short-term dynamic conflictbased psychotherapy on the subject of communication on improvement gastrointestinal symptoms and coping strategies in patients with functional indigestion. J Clin Psychol. 2011;3(4):1-13.

29. Kramer U, Pascual-Leone A, Despland JN, de Roten Y. One minute of grief: emotional processing in short-term dynamic psychotherapy for adjustment disorder. J Consult Clin Psychol. 2015;83(1):187-98. doi: 10.1037/a0037979.

30. Bourke ME, Grenyer BF. Psychotherapists' response to borderline personality disorder: a core conflictual relationship theme analysis. Psychother Res. 2010;20(6):680-91. doi: 10.1080/10503307.2010.504242.

31. Meganck R, Desmet M. The differential efficacy of supportiveexpressive and cognitive-behavioral interventions in dependent and self-critical depressive patients (GPS): findings from the Ghent Psychotherapy Study. In: 50th Annual International Meeting of the Society for Psychotherapy Research. Society of Psychotherapy Research (SPR); 2019. p. 71.

32. Kashanaki H, Aslani S. Review of intensive short-term dynamic psychotherapy: theory to practice. Rooyesh-e-Ravanshenasi Journal (RRJ). 2018;7(8):225-60. [Persian].

33. McCarthy KS, Zilcha-Mano S, Barber JP. Process research in psychodynamic psychotherapy: interventions and the therapeutic relationship. In: Kealy D, Ogrodniczuk JS, eds. Contemporary Psychodynamic Psychotherapy. Academic Press; 2019. p. 75-88. doi: 10.1016/b978-0-12-8133736.00005-2. 